and response to anti-tuberculous therapy (ATT). We test the hypothesis that FDG-PET CT imaging might be useful in OTB patients, most of whom describe visual symptoms as the presenting feature of $\mathrm{TB}$, without respiratory or constitutional symptoms. Patients were identified by analysing referrals between a tertiary referral centre for ophthalmology and its regional TB service, over a five year period, with data collection continuing prospectively. Additional cases were identified from our region's TB Register, where the eye(s) had been recorded as an extra-pulmonary site of TB. As part of TB screening, all patients had a chest X-ray (CXR) and interferon gamma release assay (with or without Tuberculin Skin Test). The TB medical team then assessed whether thoracic CT was indicated, to identify lymphadenopathy for endobronchial sampling, or an FDG-PET scan to look for avid thoracic or extrathoracic lymph nodes. In 40 patients, CXRs were essentially normal in two thirds and reported as abnormal, but not indicative of pulmonary $\mathrm{TB}$, in one third. Thoracic CT in 15 patients demonstrated abnormal features in 8 , half of whom went on to have endobronchial sampling. FDG-PET scans in 18 patients demonstrated avid nodes in 12: thoracic in 8 and extra-thoracic in 7 (cervical, axillary, pancreatic and inguinal). Overall, FDG-PET directed additional endobronchial sampling in 4 patients without enlarged thoracic nodes on conventional CT and ultrasound-guided biopsy of extra-thoracic sites in 7 patients, 2 of which subsequently demonstrated TB in culture. We describe a highly phenotyped cohort of OTB patients. There are currently no published series utilising FDG-PET CT scanning as a routine part of the investigation strategy in this condition. Whilst OTB treatment remains empirical in many cases, our preliminary Results indicate that FDG-PET is a useful imaging modality for some patients and has a potential additional yield in subclinical TB over thoracic CT imaging, allowing activity to be detected in normal-sized thoracic nodes and also extra-thoracic sites.

\section{P11 HOW EXPERT IS THE XPERT MTB/RIF FOR DRUG SUSCEPTIBLE TUBERCULOSIS?}

${ }^{1} \mathrm{JB}$ Mullerpattan, ${ }^{2} \mathrm{RB}$ Banka, ${ }^{1} \mathrm{~A}$ Khillari, ${ }^{1} \mathrm{~S}$ Ganatra, ${ }^{1} \mathrm{ZF}$ Udwadia, ${ }^{1} \mathrm{C}$ Rodriques. ${ }^{1}$ P.D. Hinduja National Hospital and Medical Research Centre, Mumbai, India; ${ }^{2}$ Norfolk and Norwich University Hospital, Norwich, UK

\subsection{6/thoraxjnl-2017-210983.153}

Background The Xpert MTB/RIF is a point of care, fully-automated diagnostic molecular test that simultaneously detects tuberculosis and rifampicin drug resistance which is used as a surrogate for multidrug resistant tuberculosis (MDR-TB). However, not much is known about susceptibility to other anti-TB drugs in patients having Rifampicin susceptibility on the Xpert. The Indian National Tuberculosis Programme recommends isoniazid and rifampicin in the continuation phase as part of category I first line therapy. However, in cases with isoniazid monoresistance, which may be missed by the Xpert $\mathrm{MTB} /$ Rif, this would lead to rifampicin monotherapy and possible amplification of resistance. Hence it is essential to know the background rates for isoniazid resistance, before deciding on the drugs to be used in the continuation phase.

Aim To analyse drug susceptibility patterns (DST) in patients with rifampicin susceptibility.

Methods This study was carried out at the microbiology laboratory of PD Hinduja National Hospital, Mumbai, India. All Mycobacterium tuberculosis (MTB) isolates who underwent 14 drug susceptibility testing (DST) between December 2015November 2016 were analysed retrospectively. All drugs were tested at WHO defined critical concentrations (CC).

Results Of a total of 2750 samples undergoing 14 drug DST, 1383 (50.29\%) were rifampicin susceptible. Among the rifampicin susceptible isolates, the most common resistance was observed to Isoniazid (INH) in 182 (13.1\%) samples of which $127(9.18 \%)$ were isoniazid monoresistant. Ofloxacin resistance was seen in $55(3.97 \%)$ samples; of these $33(2.38 \%)$ were also moxifloxacin resistant at CC of $0.5 \mathrm{mcg} / \mathrm{ml}$. Polydrug resistance was seen in $71(5.1 \%)$ isolates; of these the most common cross resistance involved INH and Ethionamide seen in $55(3.97 \%)$ isolates. Concomitant INH and fluoroqiunolone resistance was seen in $11(0.8 \%)$ isolates. Concomitant resistance to $\mathrm{INH}$, Pyrazinamide and Ethambutol was seen in 2 isolates.

Conclusion Complete DST, when available, can identify polydrug resistance and help design a more effective regimen. Increased access to Line probe assays would identify isoniazid resistance even in patients showing rifampicin susceptibility on the Xpert MTB/Rif. With high rates of INH resistance observed (13.1\%) in our setting, it may be prudent to include Ethambutol in the continuation phase to avoid rifampicin monotherapy.

\section{Asthma: airways and antibodies}

\section{P12 SUPPRESSION OF FRACTIONAL EXHALED NITRIC OXIDE WITH DIRECTLY OBSERVED INHALED CORTICOSTEROID THERAPY: IS IT A USEFUL TEST IN ROUTINE CLINICAL PRACTICE?}

${ }^{1} \mathrm{~S}$ Faruqi, ${ }^{1} \mathrm{~J}$ Thompson, ${ }^{1} \mathrm{~K}$ Watkins, ${ }^{1} \mathrm{H}$ Cummings, ${ }^{1} \mathrm{~N}$ Jackson, ${ }^{1} \mathrm{~A}$ Prakash, ${ }^{2} \mathrm{MG}$ Crooks. ${ }^{1}$ Hull and East Yorkshire Hospitals NHS Trust, Hull, UK; ${ }^{2}$ Hull York Medical School, Hull, UK

\subsection{6/thoraxjnl-2017-210983.154}

Introduction Measurement of fractional exhaled nitric oxide (FeNO) is an easy to perform non-invasive test and surrogate marker of eosinophilic airway inflammation. It has been suggested that suppression of FeNO following a week of directly observed inhaled corticosteroid (ICS) therapy provides objective evidence of non-adherence. Though guidelines for the prescription of novel steroid sparing agents recommend this strategy as part of evaluation, it has not been rigorously evaluated in routine clinical practice. We report the outcomes of using this strategy as part of standard clinical care in our centre.

Methods Consecutive consenting patients with FeNO levels greater than $45 \mathrm{ppb}$ on two occasions who were adherent to prescribed therapy on the basis of clinical history, and meeting criteria for initiation of biological therapy undertook directly observed ICS therapy - supervised (DOTS) over 8 days. In this, patients existing ICS/LABA combination inhalers were changed to once daily fluticasone/vilanterol (Relvar 184/22). Inhaler technique was taught by a specialist nurse and inhaled therapy taken under direct supervision (in person or remotely via Skype). FeNO, spirometry, eosinophil count and the Asthma Control Questionnaire -7 (ACQ-7) were recorded on the first and last days. FeNO was also recorded on day 4. Data are presented as mean $\pm \mathrm{SD}$.

Results Sixteen subjects ( 7 males, age $43 \pm 18$ years) completed assessments (1 did not have day 4 FeNO value). All but one 


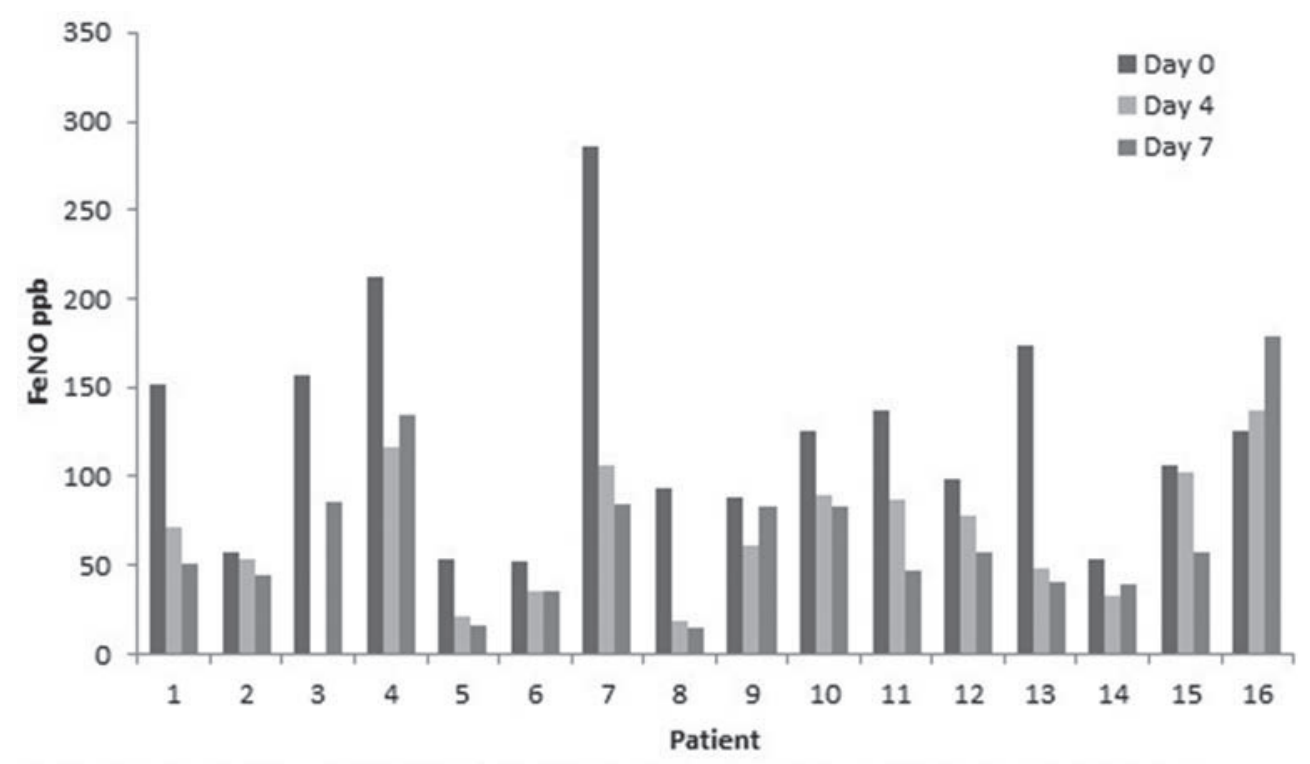

Abstract P12 Figure 1 FeNo values are depicted on the $Y$ axis and individual patients on the $X$ axis. Individual bars from left to right for a single subject depict FeNO values at days 0,4 and 7 respectively.

had $\geq 80 \%$ prescription pick-up rates checked with GP records. FeNO levels decreased from $121 \pm 63 \mathrm{ppb}$ to 71 $\pm 36 \mathrm{ppb}$ at day 4 and $66 \pm 43 \mathrm{ppb}$ at day 8 (p<0.01). 10 subjects had significant FeNO suppression by day 4 (figure 1). ACQ-7 score improved from $2.7 \pm 1.2$ to $1.7 \pm 1.1 \quad(\mathrm{p}<0.01)$. Eosinophil count $(0.65 \pm 0.51)$ and percent predicted FEV1 (79 $\pm 19)$ did not change significantly.

Conclusion Combining the use of once a day therapy with remote assessment using appropriate technology, FeNO suppression is a feasible objective test of adherence in the routine clinical setting. Despite appropriate refill collection rates over half of subjects were identified as non-adherent to inhaled therapy. Although these individuals did not commence biological therapy, improvements have been sustained. We recommend routine use of this assessment in severe asthma services.

\section{P13 BRONCHIAL THERMOPLASTY MAINTAINS A LONG-TERM REDUCTION IN PERIPHERAL BLOOD EOSINOPHILS IN SEVERE ASTHMA}

${ }^{1} \mathrm{~K}$ Hince, ${ }^{1} \mathrm{U}$ Holmes, ${ }^{1} \mathrm{G}$ McCumesky, ${ }^{2} \mathrm{D}$ Ryan, ${ }^{1} \mathrm{RM}$ Niven. ${ }^{1}$ University Hospital of South Manchester, Manchester, UK; ${ }^{2}$ Beaumont Hospital, Dublin, Ireland

\subsection{6/thoraxjnl-2017-210983.155}

Introduction Severe Asthma, characterised by persistent symptoms despite maximal medical therapy, represents $5 \%$ of asthma cases. Bronchial Thermoplasty (BT) is a novel therapy where radiofrequency thermal energy is applied to airways distal to the main-stem bronchi, permanently reducing airway smooth muscle mass. It is unknown whether treatment of smooth muscle hypertrophy impacts persistently upon systemic signs of allergic inflammation. Peripheral blood eosinophils (PBEs) are a marker of inflammation in asthma. We have previously shown that $\mathrm{BT}$ modifies signs of $\mathrm{Th}_{2}$ inflammation as measured by PBEs and therefore wanted to ascertain whether this persisted over time?

Method We reviewed the 13 consecutive Severe Asthma cases treated with BT who were included in the initial analysis. Serial PBEs measured up to 3 years post BT were compared to those in the year proceeding BT. Blood eosinophil levels taken peri-procedure were excluded from analysis due to escalated steroid therapy at this time.

Results Figure 1 demonstrates the absolute values of PBE for each patient during the 3 time periods (year before BT, Year immediately after BT and third year post BT). The group mean in year 3 has remained suppressed compared to baseline (year 3; 0.17 baseline $\mathrm{p}=0.0035$ ).

Conclusion Previous findings showed that Severe Asthma patients undergoing BT had a significant reduction in average peripheral blood eosinophil levels from baseline. The data shows overall the group of 13 patients continue to remain eosinophil supressed supporting the possibility of a long term eosinophil suppressive impact of BT.

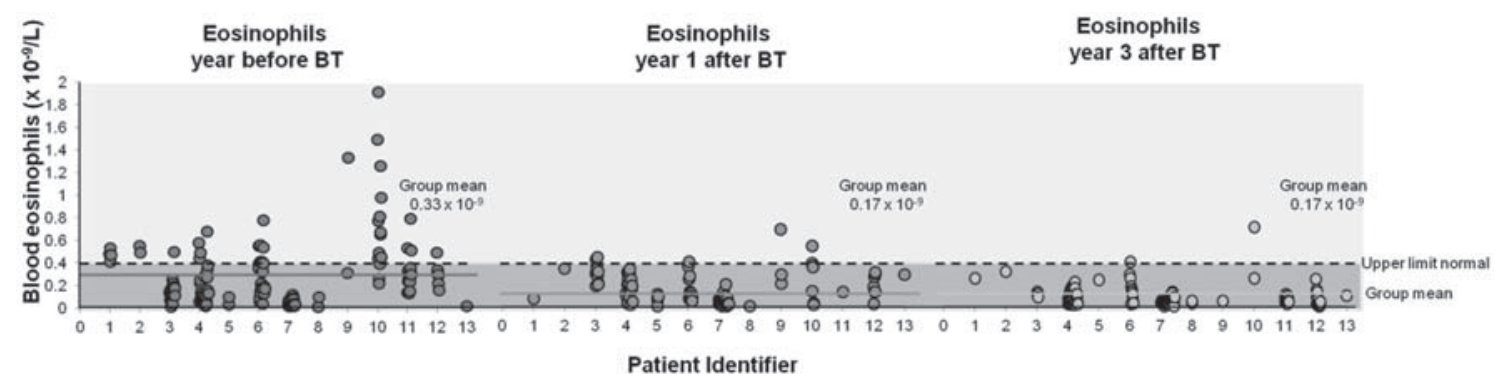

Abstract P13 Figure 1 Persistent suppression of eosinophils 3 years following bronchial thermoplasty (BT). 